\title{
RESEARCH ON THE DIGITAL SIMULATION FOR THE WHOLE PROCESS OF MARS EXPLORATION
}

\author{
Liang Lyu, Qing Xu*, Yang Zhou, Shuai Xing, Wanjie Lu, Yinghao Zhao \\ Zhengzhou Institute of Surveying and Mapping, 450052, Zhengzhou, China-13937169139@139.com
}

\author{
Commission II, ICWG III/II
}

KEY WORDS: Digital simulation; Mars exploration; Sino-Mars; Time-space benchmark; Activity object model

\begin{abstract}
:
China has paid considerable attention to space exploration and made great strides in the field. The first Chinese Mars Exploration Mission will be carried out in 2020. Digital simulation has been proved to be an effective and efficient means for planning and deduction in many fields. Thus, it was introduced for the Mars exploration in this paper and key technologies was researched above three aspects. First of all, complicated time-space benchmark was combed to support the interplanetary simulation. Secondly, the multi-resolution pyramid model and indexing strategy were adopted to preprocess the geographical environment data, which ensured the efficiency of data loading, browsing, and querying. Then, the activity objects were abstracted and modelled based on four aspects, including property, ephemeris, geometry, and behavior. Therefore, a digital simulation system, called Sino-Mars, was developed. The architecture of SinoMars consists of five layers, including data collection, data processing, scenario modelling, visualization and application layer. Using the Chinese Mars Exploration Mission slated for 2020 as an example, we demonstrated the capabilities of Sino-Mars for data integration, visualization, process deduction, and auxiliary analysis.
\end{abstract}

\section{INTRODUCTION}

Mars, as the most potential planet for life besides the earth in the solar system, has become the focus of deep space exploration in various countries after the moon. Since the 1960s, mankind has carried out dozens of Mars exploration missions. Many orbiters, landers and rovers had been launched e.g. mars global surveyor (MGS), mars express (MEX), Viking-1, Spirit, and Curiosity and son on(Murchie et al., 2016). China plans to launch own Mars probe aboard a Long March-5 carrier rocket autonomously by 2020. Despite hardware such as high-thrust rocket, detector and sensor are of growing sophistication and various types of communications, navigation, landing programs are increasingly improved, the success rate of Mars exploration missions is still less than $50 \%$ so far due to factors such as the long distance, the harsh environment and the complicated surface topography. With the development of computer technology, using digital simulation for not only planning large-scale space activities like Mars exploration in advance, but also expressing and sharing massive Mars science data afterwards has become an effective means and a hot topic.

It has been a long time since the people studied the earth with the help of digital simulation. The term Digital Earth, a digital mirror of the real world, can be traced to U.S. Vice President Al Gore in 1998 and is a typical representative. It plays a role in many aspects as a geoportal, a visualization service, a platform for simulation and prediction, a source of unprecedented spatial and temporal resolution, and a technology fully integrated into human activities(Goodchild, 2012). Platforms for example Google Earth (Google, 2017a), World Wind (NASA, 2017c), ArcGIS Earth (ESRI, 2017) and GeoGlobe (Jianya et al., 2010) have been developed and provided a new vision to learn the earth. Further, some digital visualization platforms like Google Mars (Google, 2017b), JMARS (ASU, 2017), Moonworld (Wood et al., 2010) and ADVISER ((Head et al., 2005; Huffman et al., 2011) have been developed and could support data representation and collaborative simulation. But they are often limited to a single planet and lack the capability to simulate the entire process of complex space-based activities, for instance, Mars exploration. Owing to the specialization of space science, there are not many mature aerospace mission simulation platforms so far. Systems Tool Kit (STK) (AGI, 2016) is a leading commercial software used for analysis in the aerospace field and space exploration simulation, including design, testing, launch, operation, and task application. In addition, World Wind(NASA, 2017c), General Mission Analysis Tool (GMAT) (NASA, 2017b), SaVi (Wood, 2017), 3DView (Génot et al., 2017), and Cosmographia (NASA, 2017a) could offer certain capabilities for activity simulation and scientific data visualization. However, these aforementioned platforms mainly focus on space activity simulation and have inadequate features for the analysis and visualization of a planet's surface information. As we all know, information of topography and geology is important for landing and touring, so the capacity of integration and analysis of geographical data is indispensable too. To the best of our knowledge, in China, there is no mature software similar to STK. Thus, a digital simulation means that can support China's future interplanetary space exploration missions is required.

Therefore, in this study, a digital simulation system called SinoMars was developed for the virtual visualization of space from Earth to Mars. It can be regarded as the distillation of virtual geographical environments (VGEs) (Lin et al., 2015; Lin and Chen, 2015; Lin et al., 2013; Lü, 2011) and will be helpful for the improvement of public Mars cognition. What is more, the simulation for the whole process of Mars exploration and the access and analysis of geological information can be supported with the help of this system. The architecture of the paper is organized as follows. Section 2 illustrates the key technologies including time-space reference, data organization and preprocessing and design of the visualization engine. The architecture of Sino-Mars is described in Section 3. The capability of Sino-Mars is verified by the case of Mars exploration simulation in China; the information regarding this is presented in Section 4. Finally, the conclusions are described in Section 5 . 


\section{KEY TECHNOLOGIES}

\subsection{Time-Space Benchmark}

Mars exploration is an interplanetary activity involving the earth and Mars. Accurate space-time benchmark is the foundation for the simulation, which are maintained by many specialized research institutes such as IAU (2017); IERS (2013); IUGG (2017). Because of the different sources of related data and means of obtaining this data, the spatial-temporal coordinate format of data and model interfaces vary widely. Therefore, it is extremely essential to clarify the time-space benchmark and perform mutual conversions ahead of time.

\subsubsection{Time Benchmark}

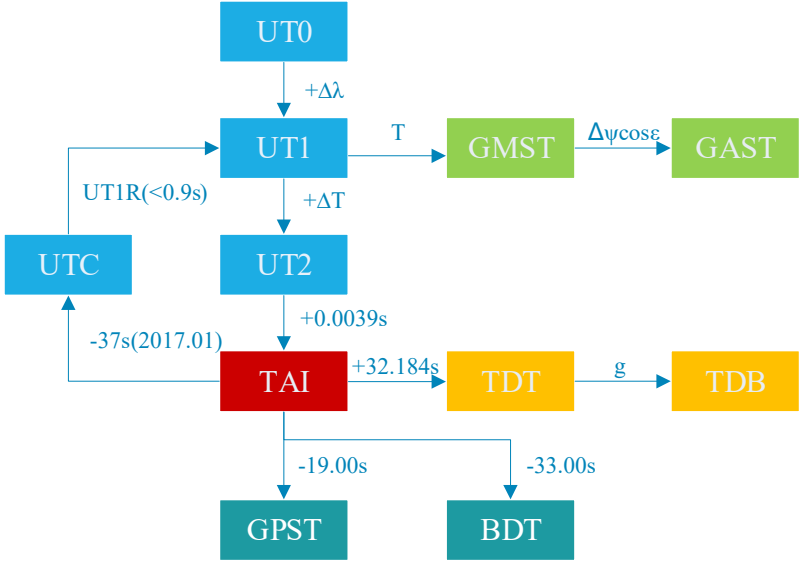

Figure 1. Time benchmark composition

The development of the time system has been guided by the pursuit of higher accuracy. The earliest definition of time derives from observations of the sun and stars. At present, the current time system primarily consists of the Sidereal Time (ST), Universal Time (UT), Ephemeris Time (ET), and International Atomic Time (TAI). In addition, other time benchmarks are defined such as Coordinated Universal Time (UTC), Global Positioning System Time (GPST), and BeiDou Navigation Satellite System Time (BDT) etc. for convenience. Figure 1 shows their correlation relationship and Petit et al. (2010) describes their sources, definitions and conversions. The simulation of Mars exploration involves multiple time systems. For example, Barycentric Dynamical Time (TDB) is widely used in the calculation of solar planets position, UTC is the most common time format in the earth, and TAI has become the standard of navigation satellite systems. The Standards of Fundamental Astronomy (SOFA) Library for ANSI C (Hohenkerk, 2011) are used to deal with the conversion between time systems.

\subsubsection{Space Benchmark}

Different from the common human activities, Mars exploration belonging to the one at the solar system scale needs to be supported by a coordinate system with a larger scale. The space benchmark primarily includes the International Celestial Reference System (ICRS), Mars Centroid Reference System (MCRS), International Terrestrial Reference System (ITRS), Rover Coordinate System, Station Coordinate System, and Satellite Coordinate System, as shown in

Figure 2. Most of them are composed of several forms. For example, different centers determine different celestial reference systems, such as heliocentric, Mars and geocentric celestial reference systems. Mars exploration refers to two planets, orbiters, landers and rovers. Accordingly, ITRS, PCRS, Satellite coordinate system, etc. and conversions between them are needed, which can be achieved from Petit et al. (2010); Xu and Xu (2013); Zhang et al. (2012). In this paper, the conversion between reference system in Sino-Mars is accomplished with the help of SOFA Library for ANSI C (Hohenkerk, 2011) as well.

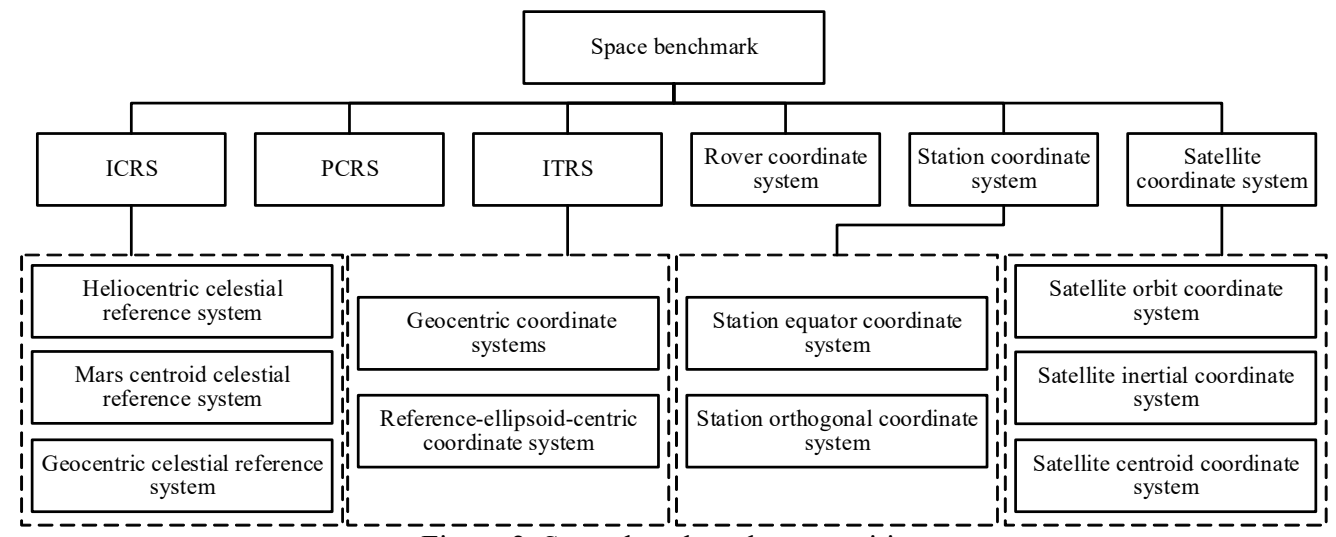

Figure 2. Space benchmark composition

\subsection{Organization and Anticipation of Geographical Environment Data}

Geographical environment data is the basis of landing area selection and reconnaissance by Mars car. In order to improve the efficiency of data loading, rendering and querying, the strategy of multi-resolution pyramid model is generally adopted by most visualization and simulation platforms (Bo et al., 2013; Chen et al., 2013). In our study, we use the geographic data pyramid system of WorldWind. The projected images and digital elevation model (DEM) data were processed using layering and blocking technologies (Figure 3).
From the first layer which was divided into $5 \times 10$, the lower layer was segmented by quadtree on the basis of upper layer. Then the resolution of images and DEM increased along with the laying level. The pixel size of pyramid tile was set to 512 (both width and height). The row increased from $90^{\circ} \mathrm{S}$ to $90^{\circ} \mathrm{N}$ and the column increased from $180^{\circ} \mathrm{W}$ to $180^{\circ} \mathrm{E}$. The row and column number (Row, Col) at the coordinate (Lon, Lat) of Ln level could be calculated using the equation (1). Three-level storage with the naming convention were used to index the tile, 'Index: \Data Folder $\backslash$ Data Set Name $\backslash L n \backslash R o w \backslash$ Col.abc', which is convenient for querying and loading. 


$$
\left\{\begin{array}{l}
\text { Row }=\left\lfloor\frac{(\text { Lat }+90) \times 2^{n}}{36}\right\rfloor \\
\text { Col }=\left\lfloor\frac{(\text { Lon }+180) \times 2^{n}}{36}\right\rfloor
\end{array}\right.
$$
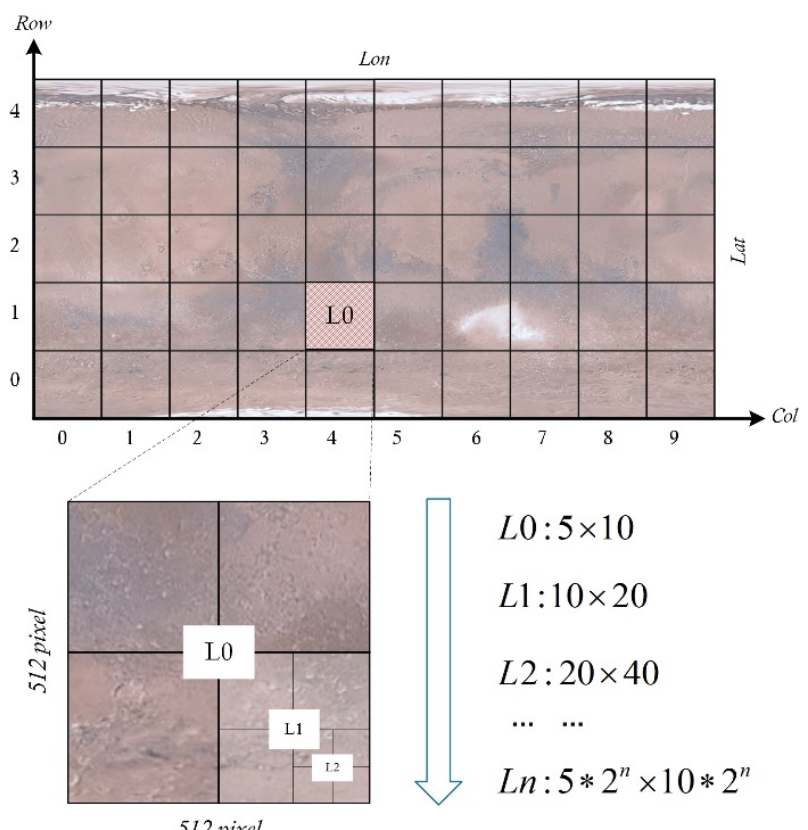

512 pixel

Figure 3. Geographic data segmentation and index method

Besides images and DEM, there are large amount of named features including Craters, Planitias, Dorsums, Vallis and so on. The point region (PR) quadtree index (Sperber, 2017) was created based on the location coordinates and recorded using the XML file, which improved the efficiency of querying and browsing.

\subsection{Activity Object Model}

Except for the geographical environment, the launch vehicle, probe and Mars car are integral components of Mars exploration, which are called activity objects. They refer to the targets of direct or indirect participation in space activities. The description of an activity object includes its position in the space-time coordinate system, the shape and attitude, and the attribute information.

As is shown in Figure 4, a unified activity object model was designed, which consists of Property, EphemerisList, Geometry, and BehaviorList. Property contains descriptions such as the name, ID, category, type and capacity of the object, which is the fundamental information. The design of trajectory is the very important work in the Mars exploration and it determines if the probe can reach Mars at the right time and position. Thus, the simulation in the paper should support the flexible loading of the track. EphemerisList is designed for this objective. As we all know, the whole process of Mars exploration comes down to multiple manoeuvres and each of them has different orbit elements. Thus, EphemerisList comprises of multiple ephemeris segments and each one contains a description of the Timeline, Coordinate system, and Orbit. Timeline represents a period of time and includes start time, end time, time step and time benchmark. Coordinate system derives from the space benchmark in section 2.1.2. Orbit can be divided into four forms, Kepler, Two-Line Orbital Element (TLE), Fixed track and Realtime track. For the description of the shape, the geometry is composed of four basic types including point, line, surface and volume. It is worth noting that a complete geometry of activity may be the combination of them. To accomplish the mission the activity object needs to do a lot of actions like a robot. For example, the solar panel of probe should rotate toward the Sun and the mechanical arm of the Mars car should move to grab stones. BehaviorList is just used to describe the action of the overall and internal components of the activity object. It is also a list of behavior phases. Each phase contains a description of the timeline, action geometry, and behavior type including three types of operations (translation, rotation, and scaling).

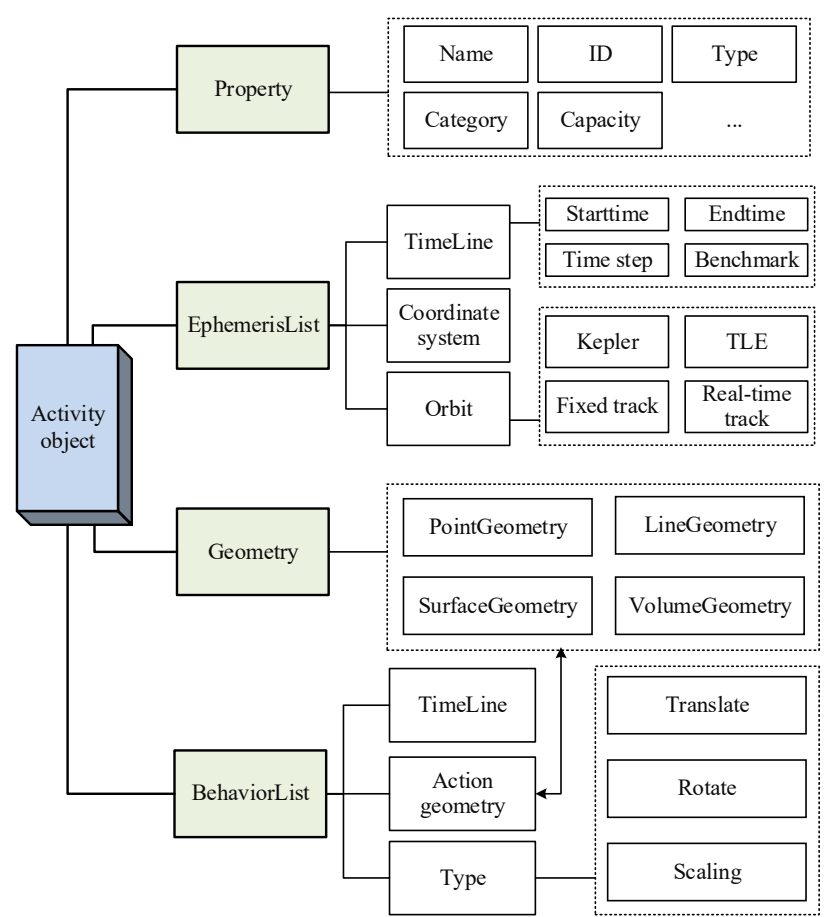

Figure 4. Unified activity object model design

\section{ARCHITECTURE OF SINO-MARS}

Base on the above key technologies, a digital simulation system called Sino-Mars was developed for the Mars exploration, which supports the visualization of geographical data, exploration process, and landing area selection, etc. Sino-Mars was developed using the programming languages $\mathrm{C}++$, Qt and OpenGL 3.0, which ensures the cross-platform features consequently.

The architecture of Sino-Mars consists of five layers, as shown in Figure 5. The data collection layer is responsible for organization and storage of entities and environment elements data, including geographic data like remote sensing images of a Mars's surface, DEM, vectors and geographical notes, tracks, 3D models and attribute information of rocket, orbiter, navigation and communication satellites. In addition, space environment elements are also considered because they have physical effect on the component of satellites. Thus, the distribution, temperature, intensity, and density of high-level atmosphere, magnetic field, ionosphere, and radiation belt are collected. The data processing layer is designed for pretreatment of data such as format conversion, accuracy interpolation, and gross error elimination of real-time and non-real-time data. Special processing strategies (e.g., pyramid and index building) for images, DEM, and note data are designed to improve the efficiency of query and visualization; processed data will be sent back to the data collection layer for storage in the case of subsequent calls. The scenario modelling layer organizes content 
that need to be expressed and analyzed using the eXtensible Markup Language (XML). The content can be saved in the local scenario file and loaded from the file again. Then, space environment model calculation, object orbit prediction, and other analyses (e.g., visibility, path analysis, crater extraction and imaging simulation) can be conducted with the support of commonly used scientific models. The visualization layer shows the distribution, form, state of entities, and environmental elements from two-dimensional (2D) and 3D perspectives, and performs multi-perspective temporal deductions with the help of viewpoint, time, and behavior control modules. In addition, the unified space-time benchmark provides a foundation for the data processing, scenario modelling, and consistency of operations in the visualization layer. The application layer as the topmost layer could support several applications, such as terrain analysis, space mission security, landing area selection, and rover route planning, among others.

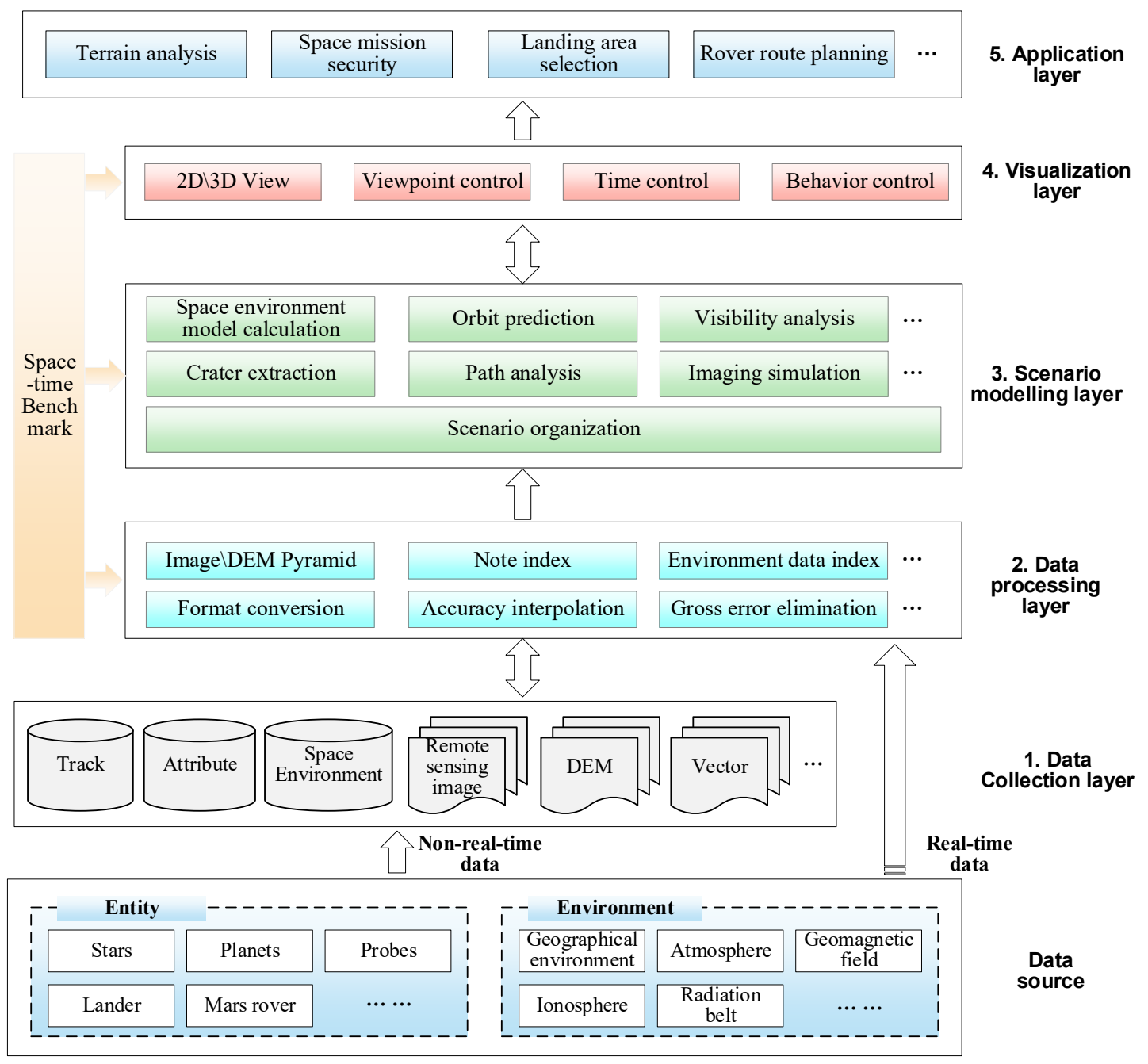

Figure 5. Overview of the Sino-Mars architecture

\section{CASE STUDY}

Considering the Chinese Mars Exploration Project 2020 as the assumed case, the entire process from launch to landing was simulated. Using the Sino-Mars, experts from different fields such as rocket launching, probe and Mars rover construction, trajectory design, measurement and control system can work together. The design scheme is continually optimized after relevant model parameters adjustment, landing area geographical analysis, and deduction results analysis, etc. The simulation was divided into three stages, as shown in Figure 6. 


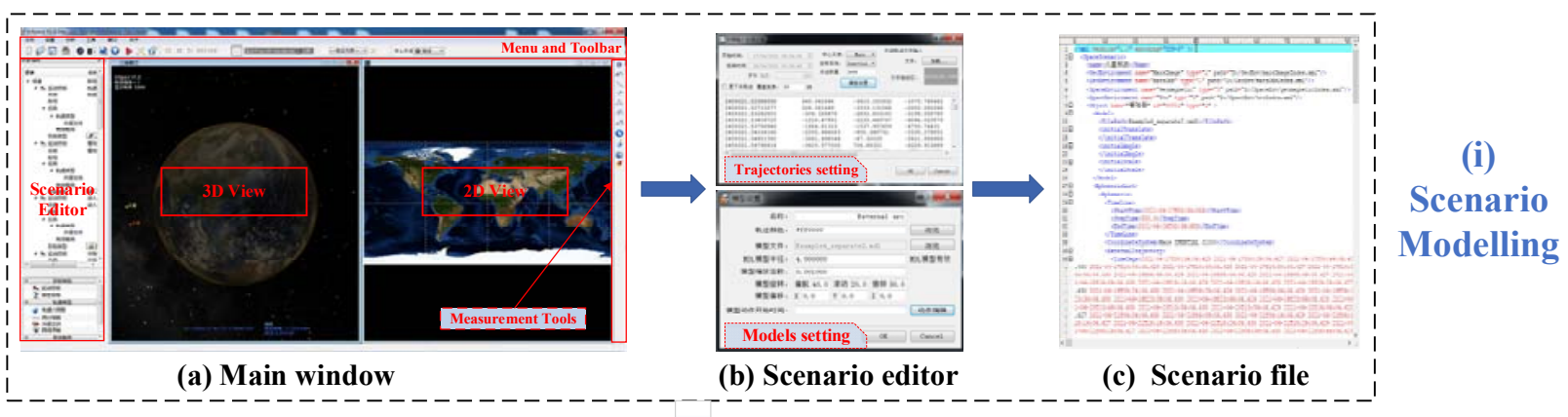

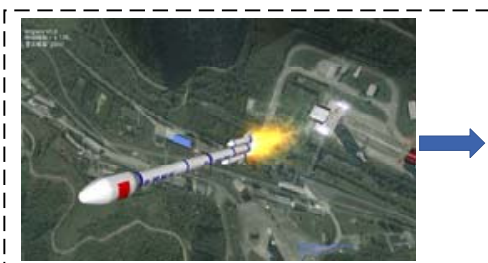

(a) Rocket launching

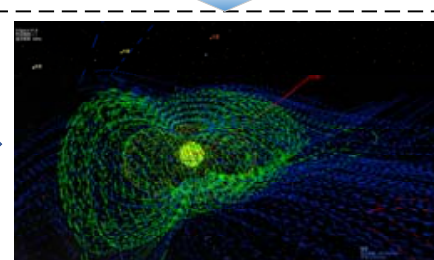

(b) Escape from the earth

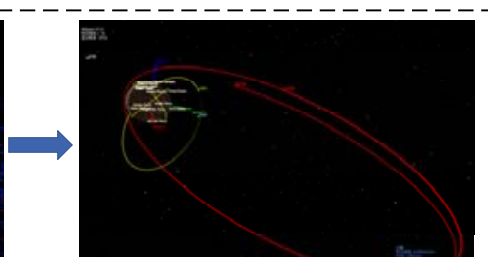

(c) Flying to Mars (ii)

Scenario

Expression

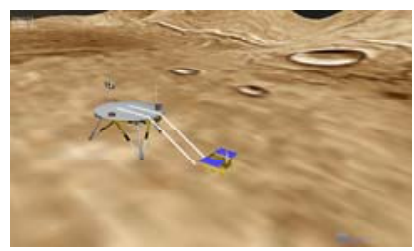

(f) Reconnaissance

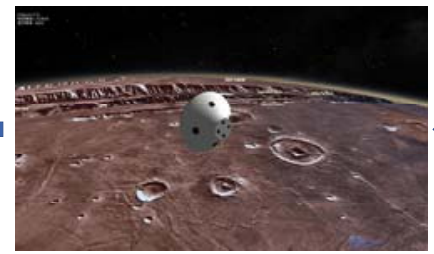

(e) Landing

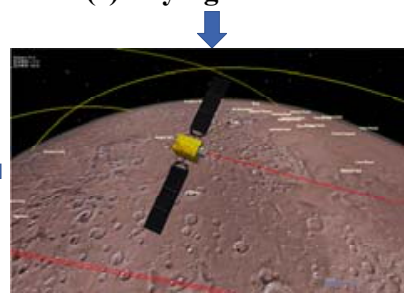

(d) Orbiting Mars

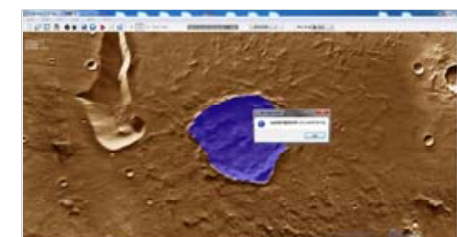

(a) Area surveying

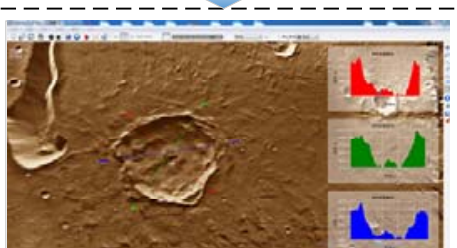

(b) Profile-map analysis

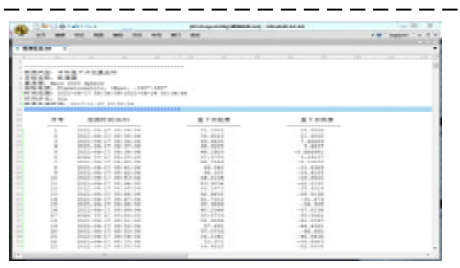

(c) Orbiter substellar points

Figure 6 . Design and simulation of the Mars exploration mission using Sino-Mars

\begin{tabular}{|c|c|}
\hline Name & Data feature \\
\hline $\begin{array}{l}\text { Mars Colorized } \\
\text { Viking } \\
\text { Mosaic(USGS, } \\
\text { 2009) }\end{array}$ & $\begin{array}{l}\text { Pixel Resolution (meters/pixel): } 232 \\
\text { Format: tif } \\
\text { Map Projection: Simple Cylindrical } \\
\text { Range: } 180^{\circ} \mathrm{W} \text { to } 180^{\circ} \mathrm{E}, 90^{\circ} \mathrm{S} \text { to } 90^{\circ} \mathrm{N}\end{array}$ \\
\hline $\begin{array}{l}\text { MOLA MEGDRs } \\
\text { (Smith et al., 2003) } \\
\text { (After conversion } \\
\text { using Global } \\
\text { Mapper) }\end{array}$ & $\begin{array}{l}\text { Pixel Resolution (meters/pixel): } 463 \\
\text { Format: tif } \\
\text { Map Projection: Simple Cylindrical } \\
\text { Range: } 180^{\circ} \mathrm{W} \text { to } 180^{\circ} \mathrm{E}, 90^{\circ} \mathrm{S} \text { to } 90^{\circ} \mathrm{N}\end{array}$ \\
\hline $\begin{array}{l}\text { All named features } \\
\text { for Mars (IAU, } \\
\text { 2009) }\end{array}$ & $\begin{array}{l}\text { Contents: Crater, Palus, Patera, and } \\
\text { Planitia, etc. } \\
\text { Format: .kmzl.shp }\end{array}$ \\
\hline
\end{tabular}

Table 1. Geographic data collected for Mars exploration simulation using Sino-Mars

(i) Scenario Modelling: This stage is the foundation of simulation for the whole process because it needs accurate design from macro to micro. Sino-Mars provides the basic virtual space environment including stellar background and planets in the solar system. The main window of Sino-Mars consists of five parts: menu and toolbar, scenario editor, 3D view, 2D view, and measurement tools. With the scenario editor, the geographic data
(Table 1) and space environment model (IGRF-12 model, IRI2012) as well as the activity objects (rocket, probe, relay satellite, Mars rover, etc.) were added. The geometry model of the probe was constructed based on design; in addition, the size and initial attitude could be adjusted. For this case, navigation experts designed and provided ephemeris information and action schedule. Subsequently, the scenario was formulated and forecasted.

(ii) Scenario Expression: The scenario simulation was carried out according to time series including rocket launch, escape from the earth, flying to Mars, orbiting Mars, landing of lander, and reconnaissance by Mars car. The menu and toolbar contain time control tools, which can control the speed of simulation and set the scenario time. The mouse can control the rotation, translation, and scaling of the viewpoint; thus, the detection process can be observed from multiple perspectives and dimensions. Conversely, the visualization results can prove the design in the stage of Scenario Modelling. If results are not appropriate, then experts can improve the process by modifying the parameters continuously.

(iii) Visual Analysis and Result Output: The geographic and spatial analysis, including Martian surface craters area measurement, topography profile analysis, and orbiter substellar points calculation, among others, were carried out before and after exploration deduction. With the help of tools containing 
point, distance, area measurement and profile analysis, we chose some landing areas and cruise routes.

\section{CONCLUSIONS}

With space exploration programs gaining popularity and increasing in relevance, the demand for digital simulation platform has increased. Further, the expansion of virtual digital earth to virtual digital universe has become imperative. As a power country, China's Mars exploration mission has been formally established and the first Mars probe will be launched in 2020. In this study, we aimed at providing a digital simulation for the Mars exploration. Firstly, we specified time-space benchmark which is more complicated compared with the activities on the earth. As the support for the surface activities on the Mars, massive geographical data most from Photogrammetry and Remote Sensing were organized using the strategy of multiresolution pyramid model. A unified model was designed to describe activity objects including their attributes, shapes, positions and actions. Then, a digital simulation system for Mars exploration, Sino-Mars was developed and the details of its structure were introduced. Further, using the simulation of the Chinese Mars Exploration Program, slated for 2020, as an example, its capabilities were verified.

The system is still in in the preliminary stage, and the following aspects will be considered in future works: (i) To improve the capacity of cooperation and interoperability, Service-Oriented Architecture (SOA) will be adopted to replace current UserOriented Structure. Then, various analysis modules using different operating systems, programming languages can be registered to the service center and provide the functions through standard and identifiable interface. (ii) Mars surface data are the main categories collected by space probes. However, geology data is crucial for the scientific research on planet evolution and origin of life. Thus, Sino-Mars will be improved to gradually increase the support for geology and underground space in the future.

\section{ACKNOWLEDGEMENTS}

We would like to thank all individuals who contributed in the development of the Sino-Mars, including the students who assisted in improving the system by testing, reporting bugs, and adding functions. We are also grateful to our users for their patience and suggestions. We thank Prof. Pingyuan Cui and the BIT navigation team for the provision of trajectory data used in this study. This work was supported by the National Program on Key Basic Research Project of China (Grant No. 2012CB720000-G), the National Natural Science Foundation of China (Grant Nos. 41701463 and 41371436), and the National Key R\&D Program of China (Grant Nos. 2016YFB0801301 and 2017YFC1200300).

\section{REFERENCES}

AGI, 2016. Systems Tool Kit https://www.agi.com/products/stk/ (10 October 2017)

\section{ASU, 2017. JMARS https://jmars.asu.edu/ (15 August 2017)}

Bo, H.G., Wu, L.X., Yu, J.Q., Yang, Y.Z., Xie, L., 2013. Rapid visualization of global image and dem based on SDOG-ESSG. Int. Arch. Photogramm. Remote Sens. Spatial Inf. Sci., XL4/W2(4), pp. 101-105.
Chen, M., Lin, H., Wen, Y., He, L., Hu, M., 2013. Construction of a virtual lunar environment platform. Int. J. Digital Earth, 6(5), pp. $469-482$.

ESRI, 2017. ArcGIS Earth http://www.esri.com/software/arcgisearth (1 December 2017)

Génot, V., Beigbeder, L., Popescu, D., Dufourg, N., Gangloff, M. Bouchemit, M., Caussarieu, S., Toniutti, J.P., Durand, J., Modolo, R., 2017. Science data visualization in planetary and heliospheric contexts with 3DView. Planet. Space Sci, 150(pp. 111-130.

Goodchild, M.F., 2012. The future of Digital Earth. Ann. Gis, 18(2), pp. 93-98.

Google, 2017a. Google Earth http://earth.google.com/

November 2017)

Google, 2017b. Google Mars https://www.google.com/mars/ (21 November 2017)

Head, J.W., Dam, A.V., Fulcomer, S.G., Forsberg, A., Rosser, G. Milkovich, S., 2005. ADVISER: Immersive Scientific Visualization Applied to Mars Research and Exploration. Photogramm. Eng. Remote Sens., 71(10), pp. 1219-1225.

Hohenkerk, C., 2011. Standards of Fundamental Astronomy. Scholarpedia, 6(1), pp. 11404.

Huffman, J., Forsberg, A., Loomis, A., Head, J., Dickson, J., Fassett, C., 2011. Integrating advanced visualization technology into the planetary Geoscience workflow. Planet. Space Sci., 59(11-12), pp. 1273-1279.

IAU, 2009. MARS_nomenclature, IAU, USGS, NASA, All named features for Mars https://planetarynames.wr.usgs.gov/shapefiles/MARS_nomencl ature.kmz

IAU, 2017. International Astronomical Union https://www.iau.org/ (18 September 2017)

IERS, 2013. International Earth Rotation and Reference Systems Service https://www.iers.org/IERS/EN/Home/home_node.html (16 September 2017)

IUGG, 2017. International Union of Geodesy and Geophysics http://www.iugg.org/ (16 September 2017)

Jianya, G., Jing, C., Longgang, X., Hanjiang, X., Huayi, W., Yandong, W., 2010. GeoGlobe:Geo-spatial Information Sharing Platform as Open Virtual Earth. Acta Geod. Cartograph. Sin., 39(pp. 551-553.

Lin, H., Batty, M., Jørgensen, S.E., Fu, B., Konecny, M., Voinov, A., Torrens, P., Lu, G., Zhu, A.X., Wilson, J.P., 2015. Virtual Environments Begin to Embrace Process - based Geographic Analysis. Trans. GIS, 19(4), pp. 493-498.

Lin, H., Chen, M., 2015. Managing and sharing geographic knowledge in virtual geographic environments (VGEs). Annal. GIS, 21(4), pp. 261-263.

Lin, H., Chen, M., Lu, G., 2013. Virtual Geographic Environment: A Workspace for Computer-Aided Geographic Experiments. Ann. Assoc. Am. Geogr., 103(3), pp. 465-482. 
Lü, G., 2011. Geographic analysis-oriented Virtual Geographic Environment: Framework, structure and functions. Sci. China Earth Sci., 41(04), pp. 733-743.

Murchie, S.L., Chabot, N.L., Buczkowski, D.L., Eng, D.A., Peplowski, P.N., Ernst, C.M., Seelos, F.P., Horanyi, M., CastilloRogez, J.C., Chmielewski, A.B., 2016. Mars-Moons Exploration, Reconnaissance, and Landed Investigation (MERLIN), Aerospace Conference, pp. 1-18.

NASA, 2017a. Cosmographia http://naif.jpl.nasa.gov/naif/cosmographia.html (1 December 2017)

NASA, 2017b. General Mission Analysis Tool https://sourceforge.net/projects/gmat/ (1 December 2017)

NASA, 2017c. NASA WorldWind https://worldwind.arc.nasa.gov/ (1 December 2017)

Petit, G., Luzum, B., Al, E., 2010. IERS Conventions (2010) https://www.iers.org/SharedDocs/Publikationen/EN/IERS/Publi cations/tn/TechnNote36/tn36.pdf;jsessionid $=0575758$ F39B0CF C0E709A864DCB9313C.live1?_blob=publicationFile\&v $=1 \quad(3$ September 2017)

Smith, D., Neumann, G., Arvidson, R.E., Guinness, E.A., Slavney, S., 2003. Mars Global Surveyor Laser Altimeter Mission Experiment Gridded Data Record, NASA Planetary Data System, MGS-M-MOLA-5-MEGDR-L3-V1.0 http://pdsgeosciences.wustl.edu/mgs/mgs-m-mola-5-megdr-13$\mathrm{v} 1 / \mathrm{mgsl} 300 \mathrm{x} / \mathrm{meg} 128 /$

Sperber, M., 2017. Quadtree and Octree, in: Shekhar, S., Xiong, H., Zhou, X. (Eds.), Encyclopedia of GIS. Springer International Publishing, Cham, pp. 1695-1700.

USGS, 2009. Viking Orbiter MDIM/MDTM/Color Mosaics, NASA AMES, Mars Colorized Viking Mosaic https://planetarymaps.usgs.gov/mosaic/Mars_Viking_MDIM21 _ClrMosaic_global_232m.tif

Wood, C.A., Reese, D.D., Ruberg, L., Harrison, A., Lightfritz, C., Avatrian, L., 2010. MoonWorld: Implementation of Virtual Lunar Exploration, the 41st Lunar and Planetary Science Conference, The Woodlands, TX, USA.

Wood, L., 2017. SaVi satellite constellation visualizer https://sourceforge.net/projects/savi/?source=recommended (1 December 2017)

Xu, G., Xu, J., 2013. Coordinate and Time Systems, Orbits: 2nd Order Singularity-Free Solutions. Springer, Berlin/Heidelberg, Germany.

Zhang, P., Xu, C., Hu, C., Chen, Y., Zhao, J., 2012. Time Scales and Time Transformations Among Satellite Navigation Systems, in: Sun, J., Liu, J., Yang, Y., Fan, S. (Eds.), China Satellite Navigation Conference (CSNC) 2012 Proceedings. Springer, Guangzhou, China, pp. 491-502. 